\title{
CONTINUED FRACTIONS \\ AND \\ PARAMETRIC GEOMETRY OF NUMBERS
}

\author{
AMINATA DITE TANTI KEITA
}

\begin{abstract}
Recently, W. M. Schmidt and L. Summerer developed a new theory called Parametric Geometry of Numbers which approximates the behaviour of the successive minima of a family of convex bodies in $\mathbb{R}^{n}$ related to the problem of simultaneous rational approximation to given real numbers. In the case of one number, we show that the qualitative behaviour of the minima reflects the continued fraction expansion of the smallest distance from this number to an integer.
\end{abstract}

\section{INTRODUCTION}

The parametric geometry of numbers, recently introduced by W. M. Schmidt and L. Summerer, is a theory which analyzes the behaviour of the successive minima of parametric families of convex bodies in $\mathbb{R}^{n}$. It provides a new approach to the problem of simultaneous approximation to real numbers by rational numbers. It was initially developed in dimension $n=3$ in [3], then extended to the general case $n \geqslant 2$ in [4], and completed in [1]. Our goal is to revisit the case $n=2$ by providing an exact description of the qualitative behaviour of the successive minima in that case. For this, we consider the family of convex bodies given by

$$
\mathcal{C}_{\xi}\left(e^{q}\right):=\left\{(x, y) \in \mathbb{R}^{2} ;|x| \leqslant e^{q},|x \xi-y| \leqslant e^{-q}\right\} \quad(q \geqslant 0),
$$

for some fixed $\xi \in \mathbb{R}$. For $j=1,2$ and $q \geqslant 0$, let $L_{\xi, j}(q)=\log \lambda_{j}\left(\mathcal{C}_{\xi}\left(e^{q}\right)\right)$, where $\lambda_{j}\left(\mathcal{C}_{\xi}\left(e^{q}\right)\right)$ denotes the $j$-th minimum of $\mathcal{C}_{\xi}\left(e^{q}\right)$, i.e. the smallest $\lambda \geqslant 0$ such that $\lambda \mathcal{C}_{\xi}\left(e^{q}\right)$ contains at least $j$ linearly independent elements in $\mathbb{Z}^{2}$. Further, we define a function $\mathbf{L}_{\xi}:[0, \infty) \longrightarrow \mathbb{R}$ by

$$
\mathbf{L}_{\xi}(q)=\left(L_{\xi, 1}(q), L_{\xi, 1}(q)\right) \quad(q \geqslant 0) .
$$

As noted by Schmidt and Summerer [3, §4], $\mathbf{L}_{\xi}$ enjoys the following properties.

2010 Mathematics Subject Classification. Primary 11J04; Secondary 11J82.

Work partially supported by NSERC (Canada). 
- Each component $L_{\xi, j}:[0, \infty) \rightarrow \mathbb{R}$ is continuous and piecewise linear with slope \pm 1 .

- If $q \in[0, \infty)$ is such that $L_{\xi, 1}$ admits a local maximum at $q$, then we have

$$
L_{\xi, 1}(q)=L_{\xi, 2}(q)
$$

- For all $q \geqslant 0$, we have $-\log 2 \leqslant L_{\xi, 1}(q)+L_{\xi, 2}(q) \leqslant 0$ (by virtue of Minkowski's theorem.)

The union of the graphs of $L_{\xi, 1}$ and $L_{\xi, 2}$ in $[0, \infty) \times \mathbb{R}$ is called the combined graph of the function $\mathbf{L}_{\xi}$, and is denoted $\boldsymbol{\Gamma}_{\xi}$. Our objective is to show that the simple continued fraction expansion of a number $\xi \in \mathbb{R}$ can be read from this graph when $\xi \in\left[0, \frac{1}{2}\right]$

Theorem. Let $\xi \in \mathbb{R}$. Then, $\boldsymbol{\Gamma}_{\xi}=\boldsymbol{\Gamma}_{\|\xi\|}$ depends only on the distance from $\xi$ to the closest integer, denoted $\|\xi\|$. Moreover, let $\left(q_{n}\right)_{0 \leqslant n<s}$ with $s \in \mathbb{N}^{\star} \cup\{\infty\}$ denote the increasing sequence of points in $[0, \infty)$ for which $L_{\xi, 1}$ admits a local maximum, and let $a_{n}$ denote for each positive integer $n<s$ the number of local maxima of $L_{\xi, 2}$ restricted to the interval $\left[q_{n-1}, q_{n}\right]$. Then it follows that $q_{0}=0$, and that the simple continued fraction expansion of $\|\xi\|$ is given by

$$
\|\xi\|= \begin{cases}{[0]} & \text { if } s=1 \\ {\left[0, a_{1}, a_{2}, \ldots, a_{s-1}\right]} & \text { if } 2 \leqslant s<\infty \\ {\left[0, a_{1}, a_{2}, \ldots\right]} & \text { if } s=\infty\end{cases}
$$

with $a_{s-1} \geqslant 2$ if $2 \leqslant s<\infty$.

In particular, the numbers $a_{n}$ are the partial quotients of $\|\xi\|$. Figure 1 illustrates this result by showing the combined graph $\Gamma_{\xi}$ (in solid lines) for several rational numbers $\xi$.

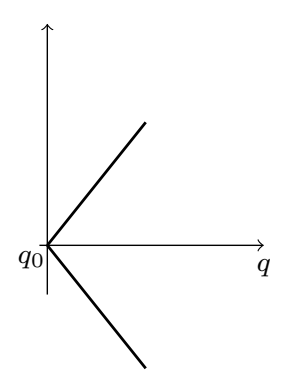
a) $0=[0]$

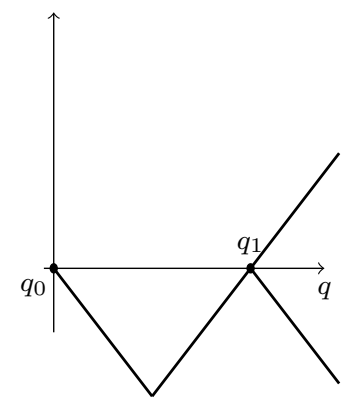

b) $1 / 2=[0,2]$

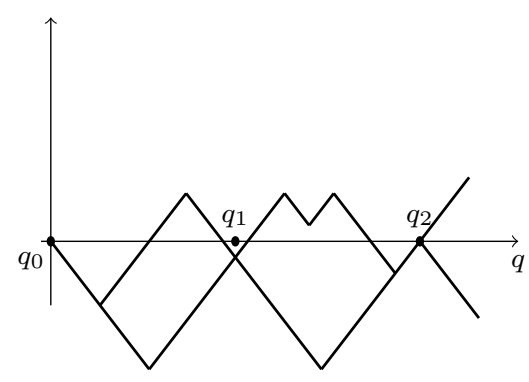

d) $3 / 7=[0,2,3]$

FIGURE 1. Examples of combined graphs. 
For the proof, fix an arbitrary $\xi \in \mathbb{R}$, and choose $m \in \mathbb{Z}$ and $\epsilon= \pm 1$ such that $\xi=m+\epsilon\|\xi\|$. The map

$$
\begin{aligned}
\phi: \quad \mathbb{R}^{2} & \longrightarrow \mathbb{R}^{2} \\
(x, y) & \longmapsto(x, \epsilon(y-m x))
\end{aligned}
$$

is an $\mathbb{R}$-vector space isomorphism which satisfies $\phi\left(\mathbb{Z}^{2}\right)=\mathbb{Z}^{2}$. Thus, for each $q \geqslant 0$, we have that

$$
\lambda_{j}\left(\mathcal{C}_{\xi}\left(e^{q}\right)\right)=\lambda_{j}\left(\phi\left(\mathcal{C}_{\xi}\left(e^{q}\right)\right)=\lambda_{j}\left(\mathcal{C}_{\|\xi\|}\left(e^{q}\right)\right) \quad(j=1,2),\right.
$$

from which it follows that $\mathbf{L}_{\xi}(q)=\mathbf{L}_{\|\xi\|}(q)$. This proves the first statement of the theorem.

For what follows, we shall suppose that $\xi=\|\xi\| \in\left[0, \frac{1}{2}\right]$. The simple continued fraction expansion of $\xi \in\left[0, \frac{1}{2}\right]$ is given by (1) for some sequence $\left(a_{n}\right)_{1 \leqslant n<s}$ in $\mathbb{N}^{\star}$, with $a_{s-1} \geqslant 2$ if $2 \leqslant s<\infty$. The $n$-th convergent of $\xi$ is the rational number

$$
\frac{P_{n}}{Q_{n}}=\left[0, a_{1}, \ldots, a_{n}\right] \quad(1 \leqslant n<s),
$$

where $P_{n}$ and $Q_{n}$ are the positive integers given by the recurrence formula

$$
Q_{n}=Q_{n-2}+a_{n} Q_{n-1} \quad \text { and } \quad P_{n}=P_{n-2}+a_{n} P_{n-1} \quad(1 \leqslant n<s),
$$

with initial values $P_{-1}=Q_{0}=1$ and $P_{0}=Q_{-1}=0$. Let $Q_{s}=\infty$ if $s<\infty$. The proof of the second part of the theorem uses the following facts (see [2, chap I]).

(i) The sequence $\left(Q_{n}\right)_{0 \leqslant n<s}$ is a strictly increasing sequence of positive integers.

(ii) The sequence $\left(Q_{n} \xi-P_{n}\right)_{-1 \leqslant n<s}$ consists of real numbers of alternating signs (except the last term which is zero if $s<\infty$ ), whose absolute values are strictly decreasing.

(iii) We have $Q_{n} P_{n-1}-Q_{n-1} P_{n}=(-1)^{n}$ for each $n \in \mathbb{N}$ satisfying $0 \leqslant n<s$.

Consider the integers

$$
Q_{n, t}=Q_{n-2}+t Q_{n-1} \quad \text { and } \quad P_{n, t}=P_{n-2}+t P_{n-1} \quad\left(0 \leqslant t \leqslant a_{n}, 1 \leqslant n<s\right) .
$$

The fractions $P_{n, t} / Q_{n, t}$ with $0<t<a_{n}$, when they exist, are called the semiconvergents of $\xi$ between $P_{n-1} / Q_{n-1}$ and $P_{n} / Q_{n}$. The following proposition relates the points $\mathbf{x}_{n, t}=\left(Q_{n, t}, P_{n, t}\right)$ to the points $\mathbf{x}_{n}=\left(Q_{n}, P_{n}\right)$ with $-1 \leqslant n<s$, as well as to the quantities $\Delta_{n}$ and $\Delta_{n, t}$ defined by

$$
\Delta_{n}=\left|Q_{n} \xi-P_{n}\right| \quad(-1 \leqslant n<s) \text { and } \Delta_{n, t}=\left|Q_{n, t} \xi-P_{n, t}\right| \quad\left(0 \leqslant t \leqslant a_{n}, 1 \leqslant n<s\right) .
$$

Proposition 1. Let $n$ be a positive integer with $n<s$. Then we have

$$
\begin{aligned}
Q_{n, 0} & =Q_{n-2}<Q_{n-1} \leqslant Q_{n, 1}<\cdots<Q_{n, a_{n}}=Q_{n}, \\
\Delta_{n, a_{n}} & =\Delta_{n}<\Delta_{n-1} \leqslant \Delta_{n, a_{n}-1}<\cdots<\Delta_{n, 0}=\Delta_{n-2},
\end{aligned}
$$


with $Q_{n, 1}=Q_{n-1}$ iff $n=1$, and $\Delta_{n-1}=\Delta_{n, a_{n}-1}$ iff $s<\infty$ and $n=s-1$. Moreover, the points $\mathbf{x}_{n, t}=\left(Q_{n, t}, P_{n, t}\right)$ with $0 \leqslant t \leqslant a_{n}$ are precisely the pairs of non-negative integers $(Q, P)$ satisfying

(5) $Q_{n-2} \leqslant Q \leqslant Q_{n}, \quad|Q \xi-P| \leqslant \Delta_{n-2}=\left|Q_{n-2} \xi-P_{n-2}\right|, \quad Q P_{n-1}-Q_{n-1} P \neq 0$.

Finally, if $s<\infty$, then there exists no pair $(Q, P) \in \mathbb{Z}^{2} \backslash\{0\}$ satisfying

$$
|Q \xi-P|<\Delta_{s-2}=\left|Q_{s-2} \xi-P_{s-2}\right|, \quad Q P_{s-1}-Q_{s-1} P \neq 0 .
$$

Due to the lack of a convenient reference, we give a succinct proof.

Proof. Since $Q_{n-2} \xi-P_{n-2}$ and $Q_{n-1} \xi-P_{n-1}$ are non-zero, of opposing signs, and since

$$
Q_{n} \xi-P_{n}=\left(Q_{n-2} \xi-P_{n-2}\right)+a_{n}\left(Q_{n-1} \xi-P_{n-1}\right)
$$

is either zero or of the same sign as $Q_{n-2} \xi-P_{n-2}$, it follows that

$$
\Delta_{n, t}=\Delta_{n-2}-t \Delta_{n-1} \quad\left(0 \leqslant t \leqslant a_{n}\right),
$$

and that $\Delta_{n, a_{n}-1}=\Delta_{n, a_{n}}+\Delta_{n-1}=\Delta_{n}+\Delta_{n-1}$. The first assertion of the Proposition then follows from properties (i) and (ii).

Let $\mathbf{x}=(Q, P)$ be a non-zero integer point satisfying (5). Since $\mathbf{x}_{n-2}=\left(Q_{n-2}, P_{n-2}\right)$ and $\mathbf{x}_{n-1}=\left(Q_{n-1}, P_{n-1}\right)$ form a basis of $\mathbb{Z}^{2}$ (by virtue of (iii)), we may write

$$
\mathbf{x}=r \mathbf{x}_{n-2}+t \mathbf{x}_{n-1} .
$$

with $(r, t) \in \mathbb{Z}^{2} \backslash\{0\}$. The third condition of (5) yields $r \neq 0$. Thus, the equality

$$
Q \xi-P=r\left(Q_{n-2} \xi-P_{n-2}\right)+t\left(Q_{n-1} \xi-P_{n-1}\right)
$$

combined with property (ii) and the second condition of (5) implies that $r t \geqslant 0$ and that $t>a_{n}$ if $r>1$. Since $Q=r Q_{n-2}+t Q_{n-1}$, then (i) allows us to conclude that $r=1$ and that $0 \leqslant t \leqslant a_{n}$.

Finally, if $s<\infty$ and $\mathbf{x}=(Q, P)$ satisfies (6), we may write $\mathbf{x}=a \mathbf{x}_{s-2}+b \mathbf{x}_{s-1}$ with $a, b \in \mathbb{Z}$ and $a \neq 0$. Since $\Delta_{s-1}=0$, we deduce that $|Q \xi-P|=a \Delta_{s-2} \geqslant \Delta_{s-2}$, contrary to the hypothesis.

For each $\mathbf{x}=(Q, P) \in \mathbb{Z}^{2}$ and each $q \geqslant 0$, we define $L_{\mathbf{x}}(q)$ to be the logarithm of the smallest real number $\lambda \geqslant 0$ such that $\mathbf{x} \in \lambda \mathcal{C}_{\xi}\left(e^{q}\right)$. A simple computation gives

$$
L_{\mathbf{x}}(q)=\max \{\log |Q|-q, \log |Q \xi-P|+q\} \quad(q \geqslant 0) .
$$

The graph of the function $L_{\mathbf{x}}:[0, \infty) \rightarrow \mathbb{R}$ is called the trajectory of the point $\mathbf{x}$. The union of these graphs for $\mathbf{x} \in \mathbb{Z}^{2} \backslash\{0\}$ primitive (i.e. with coprime coordinates) contains $\boldsymbol{\Gamma}_{\xi}$ (see [3, §4] or [1, §2]). In fact the following smaller set of points suffices.

Proposition 2. The graph $\boldsymbol{\Gamma}_{\xi}$ is covered by the trajectories of the points $\mathbf{x}_{n}$ with $-1 \leqslant n<s$ and those of the points $\mathbf{x}_{n, t}$ with $1 \leqslant n<s$ and $0<t<a_{n}$. 
Proof. Indeed, let $\mathbf{x}=(Q, P) \in \mathbb{Z}^{2}$ be primitive with $\pm \mathbf{x}$ different from all these points. Then we have $Q \neq 0$ otherwise $\mathbf{x}=(0, \pm 1)= \pm \mathbf{x}_{-1}$ against the hypothesis. Since $L_{\mathbf{x}}=L_{-\mathbf{x}}$, we may assume without loss of generality that $Q \geqslant 1$. Thus, there exists an integer $n$ with $1 \leqslant n \leqslant s$ such that $Q_{n-1} \leqslant Q<Q_{n}$, while Proposition [1] yields $|Q \xi-P| \geqslant \Delta_{n-2}$. We deduce that

$$
L_{\mathbf{x}}(q) \geqslant \max \left\{\log Q_{n-1}-q, \log \Delta_{n-2}+q\right\}=\max \left\{L_{\mathbf{x}_{n-2}}(q), L_{\mathbf{x}_{n-1}}(q)\right\},
$$

for all $q \geqslant 0$. Thus, since $\mathbf{x}_{n-2}$ and $\mathbf{x}_{n-1}$ are linearly independent, the trajectory of $\mathbf{x}$ does not contribute to the cover.

The inequalities of (3) and (44) of Proposition 1 yield

$$
L_{\mathbf{x}_{n, t}}(q) \geqslant L_{\mathbf{x}_{n-1}}(q) \quad\left(q \geqslant 0,1 \leqslant n<s, 0<t<a_{n}\right) .
$$

Since $L_{\mathbf{x}_{-1}}(q)=q \geqslant L_{\mathbf{x}_{0}}(q)$ for all $q \geqslant 0$, it follows that the graph of $L_{\xi, 1}$ is covered by the trajectories of the points $\mathbf{x}_{n}$ with $0 \leqslant n<s$, and thus that

$$
L_{\xi, 1}(q)=\min \left\{L_{\mathbf{x}_{n}}(q) ; 0 \leqslant n<s\right\} \quad(q \geqslant 0) .
$$

In particular, we have $L_{\xi, 1}(q)=L_{\mathbf{x}_{0}}(q)=-q$ near $q=0$. Thus, $q_{0}=0$ is the first local maximum of $L_{\xi, 1}$ on $[0, \infty)$. Let $n$ be a positive integer with $n<s$. The inequalities of (3) and (44) also imply that the trajectories of $\mathbf{x}_{n-1}$ and of $\mathbf{x}_{n}$ meet in a single point. To the left of this point, the trajectory of $\mathbf{x}_{n-1}$ has slope +1 , while to the right, the trajectory of $\mathbf{x}_{n}$ has slope -1 . The abscissa of this point is thus the $(n+1)$-th local maximum $q_{n}$ of $L_{\xi, 1}$ on $[0, \infty)$. It is given by

$$
q_{n}=\frac{1}{2}\left(\log Q_{n}-\log \Delta_{n-1}\right) \text {. }
$$

Moreover, $L_{\xi, 1}$ coincides with $L_{\mathbf{x}_{n-1}}$ on the interval $\left[q_{n-1}, q_{n}\right]$ (see Figure 2). Since none of the points $\mathbf{x}_{n, 0}=\mathbf{x}_{n-2}, \mathbf{x}_{n, 1}, \ldots, \mathbf{x}_{n, a_{n}}=\mathbf{x}_{n}$ is a multiple of $\mathbf{x}_{n-1}$, we further deduce from (7) that, on the same interval, we have

$$
L_{\xi, 2}(q)=\min \left\{L_{\mathbf{x}_{n, t}}(q) ; 0 \leqslant t \leqslant a_{n}\right\} \quad\left(q_{n-1} \leqslant q \leqslant q_{n}\right) .
$$

If $n \neq 1$ and $n \neq s-1$, each inequality of (3) and of (44) is strict. Thus, the trajectories of the points $\mathbf{x}_{n, t}$ with $0 \leqslant t \leqslant a_{n}$ are a part of the graph of $L_{\xi, 2}$ over $\left[q_{n-1}, q_{n}\right]$, as shown in Figure 2.b. The points at which the trajectories of $\mathbf{x}_{n, t-1}$ and of $\mathbf{x}_{n, t}$ cross for $t=1, \ldots, a_{n}$ give the $a_{n}$ local maxima of $L_{\xi, 2}$ on $\left[q_{n-1}, q_{n}\right]$, each contained in the interior of this interval. If $n=1$ and $s \geqslant 2$, the situation is illustrated by Figure 2.a. In this case, $q_{0}=0$ is the first local maximum of $L_{\xi, 2}$ on $\left[q_{0}, q_{1}\right]$. If $s<\infty$ and if $n=s-1 \geqslant 1$, then $q_{s-1}$ is the last local maximum of $L_{\xi, 2}$ on $\left[q_{s-2}, q_{s-1}\right]$, as illustrated in Figure 2.c. Finally, if $s=1$, we have that $\xi=0$ and the graph $\Gamma_{0}$ is given by Figure 1.a. In general, if $s<\infty$, we have that $L_{\xi, 2}(q)=L_{\mathbf{x}_{s-2}}(q)=q+\log \Delta_{s-2}$ for all $q \geqslant q_{s-1}$, from which it follows that $L_{\xi, 2}$ admits no local maximum on $\left[q_{s-1}, \infty\right)$. 

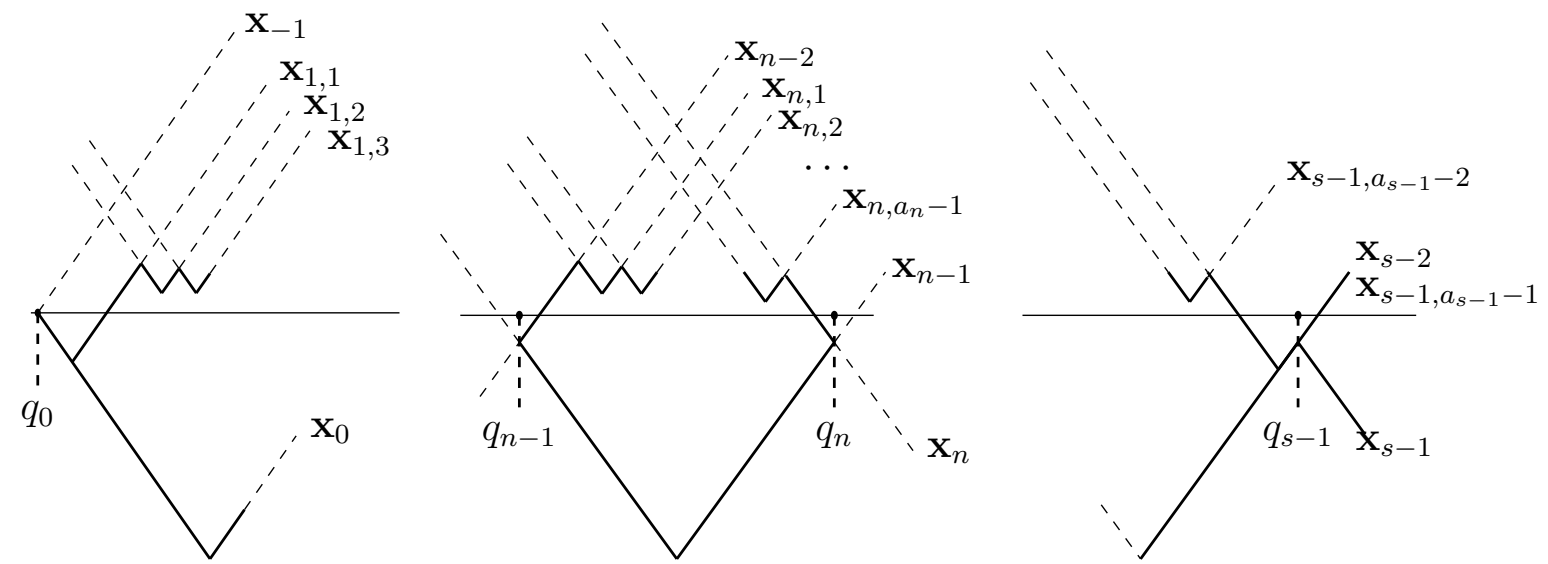

a) to the right of $q_{0}$

b) over $\left[q_{n-1}, q_{n}\right]$ if $2 \leqslant n<s-1$

c) near $q_{s-1}$ if $2 \leqslant s<\infty$

Figure 2. Combined graph $\boldsymbol{\Gamma}_{\xi}$.

\section{REFERENCES}

[1] D. Roy, On Schmidt and Summerer parametric geometry of numbers, Annals of Mathematics, to appear.

[2] W. M. Schmidt, Diophantine approximation, Lecture Notes in Math., vol. 785, SpringerVerlag, 1980.

[3] W. M. Schmidt and L. Summerer, Parametric geometry of numbers and applications, Acta Arith. 140 (2009), 67-91.

[4] W. M. Schmidt and L. Summerer, Diophantine approximation and parametric geometry of numbers, Monatsh. Math. 169 (2013), 51-104.

Département de Mathématiques, Université d'Ottawa, 585 King Edward, Ottawa, Ontario K1N 6N5, Canada

E-mail address: akeit104@uottawa.ca 